\title{
In-vitro-Diagnostik von Nahrungsmittelunverträglichkeiten: Bestimmung von allergenspezifischen Antikörpern
}

Nahrungsmittelallergien und Nahrungsmittelunverträglichkeiten gehören zu den am häufigsten von Patienten beklagten Beschwerden und es wird geschätzt, dass bis zur Hälfte der europäischen Bevölkerung hiervon betroffen sind. Zur In-vitro-Diagnostik solcher Allergien bzw. Unverträglichkeiten werden seit einigen Jahren zum Teil sehr komplexe Untersuchungssysteme angeboten, bei denen Reaktionen auf bis zu 300 verschiedene Nahrungsmittel erfasst werden. Der Stellenwert dieser Teste wird zum Teil sehr kontrovers diskutiert.

Am Anfang jeder Diagnostik einer Nahrungsmittelunverträglichkeit muss eine vollständige und gründliche Anamnese stehen. Im Rahmen dann anzuschließender Teste zum Nachweis einer spezifischen Sensibilisierung sind In-vivo-Untersuchungen zu trennen von der In-vitro-Diagnostik. Die

Die Wertigkeit der In-vivo-Untersuchungen schlieIgG-Diagnostik ist um- Ben Hautteste wie den Epikustritten-kontrollierte tan-oder Prick-Test ein sowie Studien sind rar auch Provokationsteste, bei denen Allergene z.B. oral oder nasal verabreicht werden. Im Rahmen der In-vitro-Diagnostik wird vor allem der Nachweis von spezifischen Immunglobulinen gegen Nahrungsmittel in breitem Umfang durchgeführt. Dabei ist der Nachweis von spezifischem IgE hinsichtlich seiner klinischen Relevanz kaum strittig. Hier handelt es sich um Typ-I-Allergien vom Soforttyp. Dabei liegen spezifische IgE-Antikörper gegen Proteine der Nahrungsmittel vor, die an basophile Granulozyten oder Mastzellen binden und bei erneutem Allergenkontakt zu einer Freisetzung von Mediatorsubstanzen mit nachfolgender Entzündungsreaktion führen. Nach einer entsprechenden Sensibilisierung kann es bei einem späteren Kontakt mit dem Nahrungsmittel innerhalb von Minuten bis wenigen Stunden zu einer Vielzahl von Symptomen kommen. Besonders häufig sind Schwellungen im Bereich des gesamten Verdauungstraktes. Gerade Lippen, Zunge, Gaumen und Rachen können betroffen sein. Ebenso kann es zu Erbrechen, Schmerzen im Abdomen, Meteorismus und Durchfällen kommen. Auch eine dermatologische Symptomatik z.B. im Sinne einer Urtikaria ist bekannt. Nicht in jedem Fall muss jedoch ein positiver Nachweis eines spezifischen IgE zwingend eine klinische Symptomatik zur Folge haben. Für einige klassische Allergene wie Hühnerei, Kuhmilch, Erdnuss oder Fisch ist jedoch ab bestimmten allergenspezifischen IgE-Konzentrationen eine klinische Reaktion mit einer Wahrscheinlichkeit von ca. $95 \%$ voraussagbar.

Anders ist die Situation bei der Bestimmung von spezifischen lgGAntikörpern einschließlich der Subklasse lgG 4 . Der Stellenwert dieser Diagnostik auf der Basis von lgG-Bestimmungen wird sehr kon- trovers diskutiert. So wird z.B. bei einer Insektengift-Allergie als klassischem Beispiel für eine allergische Typ-I-Reaktion unter einer spezifischen Immuntherapie im Sinne einer Hyposensibilisierung ein Anstieg des spezifischen $\lg _{4}$ als Therapieerfolg gewertet, da er meist mit einem Abfall des spezifischen lgE korreliert ist. Die Umschaltung der Produktion von IgE- auf IgG-Antikörper wird hier als Toleranzinduktion gewertet und hohe IgG $_{4}$-Titer können im Sinne »blockierender Antikörper» interpretiert werden.

Es ist bisher nicht ausreichend geklärt, warum der Nachweis von spezifischem $\lg G$ oder spezifischem $\lg _{4}$ gegen Nahrungsmittel eine klinische Bedeutung haben soll. Meist wird hier von Nahrungsmittelunverträglichkeiten gesprochen sowie von einer verzögerten, durch IgG-Antikörper vermittelten Reaktion, die innerhalb von mehreren Stunden bis zu drei Tagen nach Nahrungsaufnahme auftreten kann. $\operatorname{lgG}_{4}$ soll die Fähigkeit besitzen, selektiv an Immunzellen zu binden und bei Antigenkontakt eine Histaminfreisetzung herbeizuführen. In der komplementärmedizinischen Literatur finden sich immer wieder Arbeiten, in denen unterschiedlichste Grunderkrankungen wie entzündliche Darmerkrankungen, Neurodermitis, Migräne, psychische Leiden, chronische Müdigkeit, Adipositas, Hypertonie etc. mit lgG-vermittelten Reaktionen in Zusammenhang gebracht werden. In so genannten Anwendungsbeobachtungen fanden sich bei den vorliegenden Grunderkrankungen nach Ernährungsumstellung auf der Basis eines IgG-Nahrungsmitteltests Besserungsraten zwischen 40 und $80 \%$. In anderen Arbeiten wird die Wertigkeit der IgG-Diagnostik zur Erkennung von Nahrungsmittelallergien hingegen bestritten und es ist bekannt, dass auch bei gesunden Personen spezifische IgG-Titer zum Teil gegen eine Vielzahl von Nahrungsmitteln nachweisbar sind. Kürzlich wurde nun erstmals eine randomisierte kontrollierte Studie publiziert (Atkinson et al.: Gut 2004; 53: 1459-1464), in der gezeigt werden konnte, dass bei Patienten mit Reizdarmsyndrom eine auf der Basis eines IgG-Nahrungsmitteltests zusammengestellte Diät zu einer signifikanten Verbesserung des Beschwerdebildes im Vergleich zu einer Kontrollgruppe führte. Dies weist auf die Bedeutung IgG-vermittelter Reaktionen auf Nahrungsmittel hin.

Weiterer Forschungsbedarf ist hier in jedem Fall gegeben. Gleichzeitig muss die Vergleichbarkeit der großen Zahl angebotener Testsysteme überprüft werden. Dies betrifft sowohl die Qualität und Reinheit der Allergenpräparationen wie auch Angaben zur Reproduzierbarkeit, Sensitivität und Spezifität. Auf der Basis qualitativ hochwertiger Testsysteme müssten dann die Möglichkeiten und Grenzen der lgG-Diagnostik von Nahrungsmittelunverträglichkeiten weiter evaluiert werden.

Wolfgang Bayer 